\title{
RADIAL SYMMETRY FOR AN ELLIPTIC PDE WITH A FREE BOUNDARY
}

\author{
LAYAN EL HAJJ AND HENRIK SHAHGHOLIAN \\ (Communicated by Ryan Hynd)
}

\begin{abstract}
In this paper we prove symmetry for solutions to the semi-linear elliptic equation

$$
\Delta u=f(u) \quad \text { in } B_{1}, \quad 0 \leq u<M, \quad \text { in } B_{1}, \quad u=M, \quad \text { on } \partial B_{1},
$$

where $M>0$ is a constant, and $B_{1}$ is the unit ball. Under certain assumptions on the r.h.s. $f(u)$, the $C^{1}$-regularity of the free boundary $\partial\{u>0\}$ and a second order asymptotic expansion for $u$ at free boundary points, we derive the spherical symmetry of solutions. A key tool, in addition to the classical moving plane technique, is a boundary Harnack principle (with r.h.s.) that replaces Serrin's celebrated boundary point lemma, which is not available in our case due to lack of $C^{2}$-regularity of solutions.
\end{abstract}

\section{INTRODUCTION AND THE MAIN PROBLEM}

We use the moving-plane technique to prove symmetry for a free boundary problem, whose solutions lack $C^{2}$-smoothness up to the free boundary. Although our approach applies to more general setting, for clarity of exposition, we have considered the most simple equation, which is the following semilinear PDE

$$
\begin{cases}\Delta u=f(u) & \text { in } B_{1}, \\ u=M & \text { on } \partial B_{1},\end{cases}
$$

where $M>0$ is a constant and $B_{1}$ is the unit ball. Our departing point for regularity of solutions is $u \in\left(L^{\infty} \cap W^{1,2}\right)\left(B_{1}\right)$. Exact assumptions concerning the right hand side $f$, the solution $u$, as well as the free boundary $\partial\{u>0\}$ will be mentioned in the below. The right hand side $f(u)$ is also interpreted as $f(u) \chi_{\{u>0\}}$, and we use the notion of weak solutions

$$
\int \nabla u \cdot \nabla \varphi+f(u) \varphi=0, \quad \forall \varphi \in W_{0}^{1,2}\left(B_{1}\right),
$$

where $W_{0}^{1,2}\left(B_{1}\right)$ is the standard Sobolev space of functions, with compact support in $B_{1}$. Solutions to our problem may arise as minimizers or stationary points of the functional

$$
J(v):=\int_{B_{1}}|\nabla v|^{2}+2 F(v), \quad \text { over the class } M+W_{0}^{1,2}\left(B_{1}\right),
$$

Received by the editors December 27, 2020, and, in revised form, March 21, 2021.

2020 Mathematics Subject Classification. Primary 35B06, 35R35.

Key words and phrases. Symmetry, semilinear equations, moving plane, free boundary.

The second author was supported by Swedish Research Council. 
and where $F^{\prime}(t)=f(t)$. One may also interpret the equation in terms of viscosity, see [19]; see also the two-phase version of this problem in dimension two [13] that also can be used for possible extension of the result here to the case when $\{u<$ $0\} \neq \emptyset$.

Naturally if $M$ is large, or $f(t)$ is negative most of the time (for $t$ ), it may well happen that the dead core $\{u=0\}$ is empty. In that case the problem falls within the framework of classical theory for PDEs. Hence, the case when the dead core is non-void is more interesting and introduces some challenges.

As mentioned above, we aim at proving spherical symmetry of solutions to the problem, by employing the celebrated moving plane technique originated in A.D. Alexandrov's work [1, and cleverly used and promoted by James Serrin [15] through a new (Serrin's) boundary point lemma. The semilinear pde above was also studied extensively by Gidas-Ni-Nirenberg [11] in various forms.

When $f(t)$ is unbounded close to $t=0$ (see (1.2)), solutions to our problem are only $C^{1, \beta_{0}}$, for some $\beta_{0}>0$ depending on $f$. Hence the celebrated Serrin's boundary point lemma is not accessible anymore. We circumvent this by imposing a weak second-order asymptotic expansion for solutions close to the free boundary points; see the statement of our theorem for more details. The lack of $C^{2}$-regularity of solution 11 and hence the failure of Serrin's approach at the so-called orthogonal points (see [15]) becomes more apparent when dealing with the so-called degenerate/singular operators such as $p$-Laplacian, where in general solutions fail to be $C^{2}$, unless one assumes the gradient of the solution to be non-zero. This partly explains the reason for the lack of study for $p$-Laplacian type problems of the socalled obstacle-type; i.e. $\nabla u=0$ on the free boundary. Our approach may have some bearing on problems of similar nature.

Standing assumptions on $f(t)$. For the function $f(t)$ we assume that for some $-1<a<0, \kappa_{0}>0$ and $t_{1}>0$ (small) it has the following property:

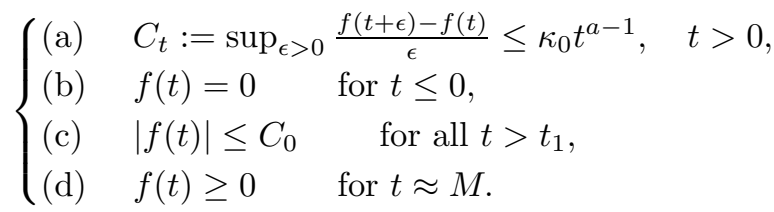

Assumption (a) is Lipschitz regularity from one side for $f$ and is needed for applying the strong maximum principle, alternatively Hopf's boundary point Lemma. The reason for introducing $-1<a<0$ is for the case when $f(t) \approx t_{+}^{a}$ close to $t=0$. When $a>0$ the solution is $C^{2, \beta_{0}}$ for some $\beta_{0}>0$ (see [18), and the problem is much softer with a straightforward proof as in the classical setting. Similarly when $a=0$, this resembles, locally, the obstacle problem, and uniqueness and non-decreasing of r.h.s. (again locally near the zero set) can be used to obtain the symmetry for the solutions.

Asymptotic property (AP). We say a solution $u$ to our problem has (AP) property if the following hold:

\footnotetext{
${ }^{1}$ The reader should know that even if we would assume the free boundary is a priori $C^{2}$ we cannot assume that the solutions are $C^{2}$, since they are not. Also the $C^{2}$-regularity of the free boundary has not been proven/disproven in the literature.
} 
(1) At any free boundary point $x^{0}$ the weak second-order asymptotic expansion holds

$$
u(x)=A_{0}\left(\left(x-x^{0}\right) \cdot \nu_{0}\right)_{+}^{\beta}+O\left(\left|x-x^{0}\right|^{2+\delta_{\beta}}\right),
$$

for some fixed $A_{0}>0$, a unit normal $\nu_{0}=\nu_{0}\left(x^{0}\right)$, and some $0<\delta_{\beta}<\beta-1$.

The real number $\beta=2 /(1-a)$, where $-1<a<0$ is the constant appearing in (1.2) or in the statement of Theorem 1

Remark 1 (Important). Property (1.3) already implies that the free boundary $\partial\{u>0\}$ is pointwise $e^{2} C^{1,|a|}$. This however does not mean that the free boundary is locally a smooth surface, since the Big-O does not provide any uniform neighbourhood for the approximation with smooth surfaces. To see the pointwise smoothness, one observes that at each free boundary point $x^{0}$ there is a unique tangent-plane $\Pi_{x^{0}}:=\left\{\left(x-x^{0}\right) \cdot \nu_{0}=0\right\}$. At any other free boundary point $z$ (close enough to $\left.x^{0}\right)$ we have

$$
0=u(z)=A_{0}\left(\left(z-x^{0}\right) \cdot \nu_{0}\right)_{+}^{\beta}+O\left(\left|z-x^{0}\right|^{2+\delta_{\beta}}\right) .
$$

Hence the distance between $z$ to this tangent-plane is

$$
\operatorname{dist}\left(z, \Pi_{x^{0}}\right)=\left|\left(z-x^{0}\right) \cdot \nu_{0}\right| \approx O\left(\left|z-x^{0}\right|^{\left(2+\delta_{\beta}\right) / \beta}\right) \leq O\left(\left|z-x^{0}\right|^{(1+|a|)}\right) .
$$

We now state our main result.

Theorem 1. For $f$ satisfying (1.2), let $u$ be a weak solution to

$$
\begin{cases}\Delta u=f(u) & \text { in } B_{1}, \\ u=M & \text { on } \partial B_{1}, \\ u<M & \text { in } B_{1},\end{cases}
$$

and suppose $f(t)=\left(t_{+}\right)^{a}, a<0$, and $t \leq t_{1}$, for some small $t_{1}>0$. We assume further that property (AP) holds, and that $\partial\{u>0\}$ is $C^{1}$. Then $u$ is spherically symmetric and $\partial_{|x|} u \geq 0$.

We remark that $f(t)=\left(t_{+}\right)^{a}$ can be replaced with $f(t)=L\left(t_{+}\right)^{a}$ for any constant $L>0$ without interfering in the proof; in that case one considers $v=u / L$ with right hand side $g(v)=f(L v) / L$.

The (uniform) $C^{1}$-regularity is only necessary in the proof of Claim $\mathbf{C}$ (see the proof in the below), when working with non-transversal touch of the moving plane with the free boundary. Otherwise the (AP) property provides us with the necessary regularity for carrying out the proof.

Theorem 1, in addition to the second-order asymptotic and $C^{1}$-regularity of the free boundary, requires no higher regularity neither for the solutions nor for the free boundary. The reason for the second-order asymptotic assumption is the super-quadratic behaviour of the solution (close to free boundary), and that the solution obviously is not $C^{2}$ up to the free boundary. Some analyses (not included in this paper) show the plausibility of a more accurate expansion, which is very natural in linearization theory of free boundary problems; see the discussion in Section 3 .

\footnotetext{
${ }^{2}$ Pointwise regularity of the free boundary $\partial\{u>0\}$ implies that for each point $z$ on this set there is a small ball $B_{r_{z}}(z)$ such that the set $\partial\{u>0\} \cap B_{r_{z}}(z)$ is trapped between two tangent

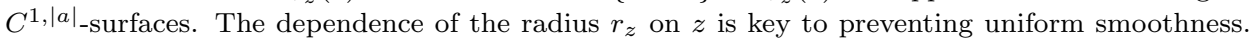
An example of such a surface can be given (in the plane) by $\partial\left\{x: x_{2}>x_{1}^{2} \sin \left(1 / x_{1}\right)\right\}$.
} 
Before closing this section we want to mention a few references that will be useful for the reader, unfamiliar with symmetry problems or free boundary techniques. For symmetry problems, besides Serrin's article [15], one can consult [11, for a more comprehensive consideration of variations of the problem. Further related results can be found in [4, 6], 8], 9], [14, [16]. It should also be mentioned that there are other applied problems that lead to free boundaries, with symmetry in focus, and where the regularity of the free boundary is a priori unknown. Let us mention one such problem, in optimization theory, with volume constraint and no a priori regularity assumptions, as done in [17. It needs to be remarked that the volume constraint usually leads to Bernoulli type free boundary; however if we replace this constraint with $L^{p}$-norm density of the solutions, then (we believe) for $0<p<1$ one may expect $C^{1, \beta_{0}}$-regularity of solutions and not $C^{2}$. Such problems may be studied with approaches suggested in this paper.

For regularity of the free boundary see [3, 5] and also [7] for basic tools. The symmetry problem for the specific form of $f(t) \approx t_{+}^{a}$ for $-1<a<0(t \approx 0)$ has not been considered in the literatures. The reason for this is the lack of $C^{2}$-regularity of solutions, since they are $C^{1, \beta-1}(\beta=2 /(1-a))$ across the free boundary and so far it is only known that the free boundary for minimizers (for the corresponding functional) are $C^{1, \alpha}$ for some $\alpha>0$, at the so-called flat points. Nevertheless, if one imposes the extra restriction so that near the set $\{u=0\}$ the solutions are local minimizers, then through a simple comparison argument one can prove monotonicity of the functional at local-level, which can be used to prove the theorem in more general setting and with almost no assumptions; see [3, Lemma 1.7] for the comparison argument.

\section{Proof OF THE MAIN THEOREM}

Since the direction can be chosen arbitrarily it suffices to show symmetry in the $x_{1}$ direction only. We will first establish some notations

$$
\begin{cases}T_{\lambda}=\left\{x \in \mathbb{R}^{n} \mid x_{1}=\lambda\right\} & \text { (the hyperplane), } \\ x^{\lambda}=\left(2 \lambda-x_{1}, x_{2}, x_{3}, \ldots\right) & \text { (the reflection of } \left.x \text { at } T_{\lambda}\right), \\ \Sigma_{\lambda}=\left\{x \mid x^{\lambda} \in B_{1} \text { and } x_{1}<\lambda\right\} & \text { (the reflection of the right half of } \left.B_{1}\right), \\ u_{\lambda}(x)=u\left(x^{\lambda}\right) & \text { (the reflection of } \left.u \text { at } T_{\lambda}\right) .\end{cases}
$$

In the setting of (2.1), we need to prove that for $\lambda=0$ we have $u_{\lambda}(x) \geq u(x)$ for all $x \in \Sigma_{\lambda}$. This will then prove that $u_{\lambda}=u$ for $\lambda=0$, for all possible rotations of the solution, that is the desired result.

We first note that by assumption (d) in (1.2), the function $u$ is a subsolution in a small interior neighborhood of $\partial B_{1}$, and by virtue of $u<M$ in $B_{1}$ and the Hopf's boundary point lemma, we get

$$
\partial_{\nu} u(y)<0, \quad \forall y \in \partial B_{1},
$$

where $\nu$ is any direction pointing into the interior of $B_{1}$ at $y$.

Now due to the strict monotonicity (2.2) for small (positive) values of $1-\lambda$ (i.e. $1 \approx \lambda<1), u_{\lambda}(x)>u(x)$ in $\Sigma_{\lambda}$. We let $\lambda_{0} \geq 0$ be the smallest $\lambda$ such that $u_{\lambda}(x)>u(x)$ in $\Sigma_{\lambda}$, for all $\left\{\lambda: \lambda_{0}<\lambda<1\right\}$. It thus follows that the level-surfaces $\{u=l\} \cap\left\{x_{1}>\lambda\right\}\left(\lambda>\lambda_{0}\right)$ will be Lipschitz graphs, where the Lipschitz norm depends on $\tau_{\lambda}$ and may blow-up exactly at $\left\{x_{1}=\lambda\right\}$. 
We next assume $\lambda_{0}>0$ (otherwise there is nothing to prove) and try to reach a contradiction by moving the plane slightly further with some $\epsilon>0$ distance to $x_{1}=\lambda_{\epsilon}:=\lambda_{0}-\epsilon>0$ and show that $u_{\lambda_{\epsilon}} \geq u$ in $\Sigma_{\lambda_{\epsilon}}$.

By minimality of $\lambda_{0}$, there are sequences $\epsilon_{i} \searrow 0, \lambda_{i}=\lambda_{0}-\epsilon_{i}$ such that $\left\{u_{\lambda_{i}}(x)<\right.$ $u(x)\} \neq \emptyset$. Define $w_{\lambda_{i}}(x)=u_{\lambda_{i}}(x)-u(x)$; then the set $D_{i}:=\left\{w_{\lambda_{i}}<0\right\}$ is nonvoid, otherwise there is nothing to prove. Define $D_{0}=\limsup _{\epsilon_{i} \rightarrow 0} D_{i}$; then $D_{0} \neq \emptyset$, unless $D_{i}=\emptyset$ for $i \geq i_{0}$ (some $i_{0}$ ). Now for $w_{\lambda_{0}}=\lim _{i} w_{\lambda_{i}}$ it follows that

$$
\nabla w_{\lambda_{0}}=0 \text { and } w_{\lambda_{0}}=0 \text { on } D_{0} \text {. }
$$

To see this, let $x^{0} \in D_{0}$, and $\left\{x^{i}\right\} \in D_{i}$, with $x^{i} \rightarrow x^{0}$. Since $D_{i}$ is an open set, for any direction $e$ there is a line segment $\left[z_{1}^{i}, z_{2}^{i}\right] \subset D_{i}$ with the end points $z_{1}^{i}, z_{2}^{i} \in \partial D_{i}$, and $z_{1}^{i}=z_{2}^{i}+t e$ for some $t$, and $x^{i}$ lying on this segment. Since $w_{\lambda_{i}}\left(z_{1}^{i}\right)=w_{\lambda_{i}}\left(z_{2}^{i}\right)=0$ and $w_{\lambda_{i}}$ is $C^{1, \alpha}$ we have $\partial_{e} w_{\lambda_{i}}\left(z_{3}^{i}\right)=0$ for some $z_{3}^{i}$ on this line segment. Now as $\epsilon_{i} \searrow 0$ we have that either $\left|z_{3}^{i}-x^{i}\right| \rightarrow 0$ or that the line segment $\left[x^{i}, z_{3}^{i}\right]$ converges to another line segment $\left[x^{0}, z^{0}\right] \in D_{0}$. In either case we have $\partial_{e} w_{\lambda_{0}}\left(x^{0}\right)=0$. Since $e$ is arbitrary we have that $\nabla w_{\lambda_{0}}\left(x^{0}\right)=0$, for $x^{0} \in D_{0}$. A similar argument shows that $D_{0} \subset\left\{w_{\lambda_{0}}=0\right\}$.

The theorem is proved if we can show that $D_{0}=\emptyset$. We remark that

$$
D_{0}=\left(D_{0} \cap \overline{\Sigma_{\lambda_{0}}} \backslash\{u=0\}\right) \cup\left(D_{0} \cap \Sigma_{\lambda_{0}} \cap\{u=0\}\right) \cup\left(D_{0} \cap T_{\lambda_{0}} \cap\{u=0\}\right) \text {. }
$$

We shall now prove that all these sets are empty. We also assume throughout the proof that $w_{\lambda_{0}} \not \equiv 0$, since $\lambda_{0}>0$. We use this tacitly in contradiction arguments in the below, when applying the comparison principle.

Claim A. $D_{0} \cap \overline{\Sigma_{\lambda_{0}}} \backslash\{u=0\}=\emptyset$.

The claim will follow if we can prove that for small values of $\epsilon_{i}$ the set $D_{i}$ must lie in $\delta_{i}$-vicinity of the free boundary $\partial\{u>0\}$, where $\delta_{i}=\delta\left(\epsilon_{i}\right) \searrow 0$. More exactly, there exists $\delta_{i}=\delta\left(\epsilon_{i}\right) \searrow 0$ such that

$$
D_{i} \subset \delta_{i}-\text { Neighborhood of }\left(\{u=0\} \cap \Sigma_{\lambda_{i}}\right) .
$$

We first notice that by (2.2) $D_{i}$ stays uniformly away from $\partial B_{1}$. Hence to prove Claim $₫$ it suffices to show that for any sequence $x^{i} \in D_{i}$ converging to $x^{0}$ in $D_{0}$, none of the following two cases hold:

(A1) $x^{0}$ is an interior point of $\Sigma_{\lambda_{0}} \backslash\{u=0\}$.

(A2) $x^{0} \in T_{\lambda_{0}} \backslash\{u=0\}$.

By the first assumption in (1.2) we have

$$
\Delta w_{\lambda_{0}}=\Delta u_{\lambda_{0}}-\Delta u=f\left(u_{\lambda_{0}}\right)-f(u) \leq c_{1}\left(u_{\lambda_{0}}-u\right)=c_{1} w_{\lambda_{0}},
$$

where $c_{1}=c_{1}(x)=\kappa_{0} u^{a-1}(x) \geq 0$, which is bounded on compact sets in $\{u>0\}$. This implies that

$$
\Delta w_{\lambda_{0}}-c_{1} w_{\lambda_{0}} \leq 0, \quad \text { in } \Sigma_{\lambda_{0}} .
$$

Since also $w_{\lambda_{0}} \geq 0$ in $\Sigma_{\lambda_{0}}$ from the strong minimum principle it follows that

$$
w_{\lambda_{0}}>0 \quad \text { in } \Sigma_{\lambda_{0}} \backslash\{u=0\},
$$

which proves that (A1) does not hold.

To prove that (A2) is not possible, we may, by virtue of (2.6), invoke Hopf's boundary point lemma, applied to $w_{\lambda_{0}}$ in a ball of radius $r_{\delta}$ for some $\delta>0$ touching at $x^{0} \in T_{\lambda_{0}} \backslash\{u<\delta\}$ we conclude that (for some $c_{\delta}>0$ )

$$
\partial_{x_{1}} w_{\lambda_{0}}\left(x^{0}\right) \leq-c_{\delta}<0
$$


contradicting (2.3). Since $\delta>0$ is arbitrary we conclude that $x^{0} \notin T_{\lambda_{0}} \backslash\{u=0\}$, and this proves Claim $\mathrm{A}$

Claim B. $D_{0} \cap \Sigma_{\lambda_{0}} \cap\{u=0\}=\emptyset$.

Suppose, towards a contradiction, that there is a point $x^{0} \in D_{0} \cap \Sigma_{\lambda_{0}} \cap\{u=0\}$. We thus have $w_{\lambda_{0}} \geq 0$ in $\Sigma_{\lambda_{0}} \backslash\{u=0\}, w_{\lambda_{0}}\left(x^{0}\right)=0$, and $\nabla w_{\lambda_{0}}\left(x^{0}\right)=0$ by (2.3). Therefore $x^{0} \in \partial\left\{u_{\lambda_{0}}>0\right\} \cap \partial\{u>0\}$, since otherwise $w_{\lambda_{0}}\left(x^{0}\right)=u_{\lambda_{0}}\left(x^{0}\right)>0$.

We also have $\Delta w_{\lambda_{0}}(x)=u_{\lambda_{0}}^{a}-u^{a} \leq 0$ in the set $\Sigma_{\lambda_{0}} \cap\{u>0\}$ and hence we can construct a harmonic barrier in the cone $K_{x^{0}} \subset\{u>0\}$, with large enough apparatus and vertex at $x^{0}$; we assume it is truncated with the ball $B_{r_{0}}\left(x^{0}\right), r_{0}>0$ small and fixed. Indeed, we let $h$ be the harmonic function in this truncated cone with zero boundary values on the surface of the cone, and $h=w_{\lambda_{0}}$ on $\partial B_{r_{0}}\left(x^{0}\right) \cap$ $K_{x^{0}}$, so that by the comparison principle $h \leq w_{\lambda_{0}}$ in $K_{x^{0}}$. From standard regularity theory (Appendix) for $u$, and hence for $w_{\lambda_{0}}$ it follows that $w_{\lambda_{0}}(x) \leq C\left|x-x^{0}\right|^{\beta}$ in $B_{r}\left(x^{0}\right) \cap\{u>0\}$. Since $\partial\{u>0\}$ is pointwis $\}^{3} C^{1}$ at $x^{0}$, we can make the opening of the cone large enough such that for some small $\epsilon>0$ we have $\sup _{B_{r}\left(x^{0}\right) \cap K_{x^{0}}} h(x) \geq$ $C r^{\beta-\epsilon}$, close to $x^{0}$ contradicting $h \leq w_{\lambda_{0}}$ in $K_{x^{0}}$.

Claim C. $D_{0} \cap T_{\lambda_{0}} \cap\{u=0\}=\emptyset$.

Since the free boundary is pointwise $C^{1,|a|}$, each boundary point $z$ has a normal vector $\nu_{z}$. With this in mind we shall now consider two cases:

(i) $T_{\lambda_{0}}$ is orthogonal to $\partial\{u>0\}$ at some point.

(ii) $T_{\lambda_{0}}$ is non-orthogonal to $\partial\{u>0\}$.

In case (i) we invoke the second asymptotic expansion (see Appendix) and will have that $w_{\lambda_{0}}=o\left(|x|^{2+\delta_{\beta}}\right)$ and is superharmonic in the set $\{u>0\} \cap \Sigma_{\lambda_{0}} \cap B_{r}\left(x^{0}\right)$.

Since at the corner point $x^{0}$ the plane is orthogonal to the free boundary, which is pointwise $C^{1,|a|}$, we may consider a rotation such that $e_{n}=\left(0^{\prime}, 1\right)$ is the normal vector to the free boundary pointing towards $\{u>0\}$. Next we let $P_{r}:=\left\{x: x_{n} \geq\right.$ $\left.x_{n}^{0}+c\left|x^{\prime}-\left(x^{0}\right)^{\prime}\right|^{1+|a| / 2}\right\} \cap B_{r}\left(x^{0}\right)$, with $c$ small such that $P_{r} \subset\{u>0\}$, for $r$ small enough.

We consider the harmonic function $h$ in $P_{r}$ with boundary values zero on $\partial P_{r} \backslash$ $\partial B_{r}\left(x^{0}\right)$, and such that $h \geq 0$ on $\partial B_{r}\left(x^{0}\right) \cap \partial P_{r}$, with $h\left(x^{0}+r e_{n}\right)=1$, say. Define further $z(x)=\left(\lambda_{0}-x_{1}\right) h^{1+\delta_{\beta} / 2}(x)$, which satisfies

$$
\Delta z=\delta_{\beta}\left(1+\delta_{\beta}\right)\left(\lambda_{0}-x_{1}\right) h^{\delta_{\beta}-1}|\nabla h|^{2}-\left(\partial_{1} h\right) h^{\delta_{\beta} / 2} \geq-\left(\partial_{1} h\right) h^{\delta_{\beta} / 2}
$$

and by symmetry we have $\partial_{1} h(x)=0$ on $P_{r} \cap\left\{x_{1}=\lambda_{0}\right\}$, and $\partial_{1} h \leq 0$ in $P_{r} \cap$ $\left\{x_{1}<\lambda_{0}\right\}$. Also, due to $C^{1,|a| / 2}$-regularity of the boundary of $P_{r}$ close to $x^{0}$, and $\partial_{1} h\left(x^{0}\right)=0$ (since $e_{1}$ is tangential direction) it must hold that $\left|\partial_{1} h\right| \leq c\left|x-x^{0}\right|^{|a| / 2}$. In conclusion we have

$$
-c \operatorname{dist}^{\epsilon_{0}}\left(x, \partial\left(P_{r} \cap\left\{x_{1}<\lambda_{0}\right\}\right)\right) \leq \Delta z(x) \leq 0,
$$

where $c>0$ is universal constant, and $\epsilon_{0}=\min \left(|a| / 2, \delta_{\beta} / 2\right)$. Let $h_{1}$ be the harmonic minorant of the superharmonic function $w_{\lambda_{0}}$ in $P_{r} \cap\left\{x_{1}<x_{1}^{0}\right\}$, so that $h_{1} \leq w_{\lambda_{0}}$ there. Next apply the boundary Harnack principle with r.h.s. as in

\footnotetext{
${ }^{3}$ Actually it is pointwise $C^{1,|a|}$ and hence also Hopf's boundary point lemma can also be applied.
} 
Theorem 3.10 in [2] for $h_{1}$ and $z$, in $P_{r} \cap\left\{x_{1}<x_{1}^{0}\right\}$ to conclude $z \leq C h_{1} \leq C w_{\lambda_{0}}$ in $P_{r / 2}$. In particular for $r_{0} \ll r$ we have

$$
c_{1} r_{0}^{2+\delta_{\beta} / 2} \leq \sup _{B_{r_{0}}\left(x^{0}\right)} z(x) \leq C \sup _{B_{r_{0}}\left(x^{0}\right)} w_{\lambda_{0}} \leq O\left(r_{0}^{2+\delta_{\beta}}\right),
$$

which obviously is a contradiction for small enough $r_{0}$.

Now we consider case (ii). We let $x^{0} \in D_{0} \cap T_{\lambda_{0}} \cap\{u=0\}$, and observe that since $T_{\lambda_{0}}$ is non-orthogonal to $\partial\{u>0\}$, and the normal vector $\nu=\left(\nu_{1}, \cdots, \nu_{n}\right)$ to the free boundary (pointing towards $\{u=0\}$ ) at points of $D_{0} \cap T_{\lambda_{0}} \cap\{u=0\}$ is pointing towards $\left\{x_{1}<x_{1}^{0}\right\}$, i.e. $\nu_{1}<0$.

Next we move the plane slightly further, and call it $T_{\lambda_{i}}=\left\{x_{1}=\lambda_{0}-\epsilon_{i}\right\}$. Let now $x^{i} \in D_{i}$ be the minimum point for $w_{\lambda_{i}}$ as described earlier. Let further $r_{i}=\left|x^{i}-y^{i}\right|$, where $y^{i} \in T_{\lambda_{i}} \cap\{u=0\}$ is the closest point to $x^{i}$. Denote also $\nu^{i}=\left(\nu_{1}^{i}, \cdots, \nu_{n}^{i}\right)$ to be the unit normal vector to the free boundary at $y^{i}$, and choose $\epsilon_{i}$ small enough such that $\nu_{1}^{i}<\nu_{1} / 2<0$. This is possible due to $C^{1}$-smoothness assumption of the free boundary.

We scale the solution according to

$$
h_{i}(x):=u\left(r_{i} x+y^{i}\right) / r_{i}^{\beta}, \quad \tilde{h}_{i}(x):=u_{\lambda_{i}}\left(r_{i} x+y^{i}\right) / r_{i}^{\beta}
$$

keeping in mind that $\nabla h_{i}\left(z^{i}\right)=\nabla \tilde{h}_{i}\left(z^{i}\right)$, where $z^{i}=\left(x^{i}-y^{i}\right) / r_{i}$. A subsequence of $h_{i}$ will converge to a global solution $h_{0}$ solving $\Delta h_{0}=h_{0}^{a}$, in the entire space, where $\nabla h_{0}\left(z^{0}\right)=\nabla \tilde{h}_{0}\left(z^{0}\right)$, where $z^{0}=\lim _{i}\left(x^{i}-y^{i}\right) / r_{i}$. Since the free boundary is smooth the limit function $h_{0}$ is a global one dimensional solution $h_{0}(x)=A_{0}\left(\left(x_{1}-\right.\right.$ $\left.\left.1, x^{\prime}\right) \cdot \nu\right)_{+}^{\beta}$; observe that due to $C^{1}$-smoothness of the free boundary $\nu^{i} \rightarrow \nu$. This along with (1.3) implies (by equating the derivative in the $x_{1}$-direction at $y^{0}$ )

$$
-\nu_{1}\left(-\nu_{1}\left(y_{1}^{0}-1\right)+\left(y^{\prime}\right)^{0} \cdot \nu^{\prime}\right)_{+}^{\beta-1}=\nu_{1}\left(\nu_{1}\left(y_{1}^{0}-1\right)+\left(y^{\prime}\right)^{0} \cdot \nu^{\prime}\right)_{+}^{\beta-1} \quad\left(\nu_{1} \neq 0\right)
$$

which is impossible, and hence the set $D_{i}$ is empty proving the claim in this case.

In conclusion the set $D_{0}=\emptyset$, and hence $\lambda_{0}=0$ and the proof of the theorem is completed.

\section{Appendix}

In this appendix we shall provide some basic facts about solutions to the problem studied in this paper. First and foremost we need to mention that solutions to problem (1.4) do exist in terms of minimizers (in an appropriate function space) of the functional

$$
\int_{B_{1}}|\nabla u|^{2}+2 F(u)
$$

where $F^{\prime}(t)=f(t)$, and the boundary value $u=M$ has been prescribed on $\partial B_{1}$.

Since we are considering more general solutions, rather than minimizers, we shall only focus on properties of solutions close to the dead core $\{u=0\}$. The regularity properties for both $u$ and the free boundary $\partial\{u>0\}$ have been studied in a few papers that we use as basic references, but we shall also explain heuristically the passage at some points that will be needed for our analysis.

The paper by Alt-Phillips [3] considers minimizes of the corresponding functional. However most of the results of their paper carry over to solutions of our problem as well. E. Teixeira [19] considers viscosity solutions and proves general regularity of solutions. Indeed, by modern techniques such as blow-up one can prove 
(by indirect method) that solutions to our problem satisfy (for positive constants $\left.c_{1}, c_{2}\right)$

$$
u(x) \leq c_{1}(d(x))^{\beta} \quad|\nabla u|(x) \leq c_{2}(d(x))^{\beta-1},
$$

where $d(x)=\operatorname{dist}(x,\{u=0\})$. E. Lindgren and L. Silvestre 12 (unpublished) proved this for weak solutions; the proof is based on blow-up and a Liouville type argument. These estimates also hold for viscosity solutions of our equations as done in [19]. M. Giaquinta and E. Giusti [10] proved the bound for minimizers.

Another important tool/property is the non-degeneracy, which states that for positive constants $c_{1}, c_{2}$

$$
c_{1} r^{\beta} \leq \sup _{B_{r}(z)} u(x), \quad \forall z \in \Gamma,
$$

whose proof is much simpler and standard and can be found in [5, Lemma 3.7 along with Remark 2.5]; the proof works also for our case with no changes. We mention this property in order to bring the attention of the reader to the fact that our solutions are not $C^{2}$ up to the free boundary.

Since the assumption of the asymptotic expansion in our main theorem is the cornerstone for the approach, we shall discuss it here at a heuristic level, but only for the regular free boundary points; similar discussion and reasoning can be made for singular points, but we shall not discuss it here. We remark that at a $C^{1}$-free boundary (or even a flat-enough free boundary) the solution $u$ should reasonably behave like a one-dimensional solution, i.e.

$$
u(x)=A_{0}\left(\left(x-x^{0}\right) \cdot \nu_{0}\right)_{+}^{\beta}+O\left(\left|x-x^{0}\right|^{2+\delta_{\beta}}\right),
$$

for some directional vector $\nu_{0}$, and a positive constant $A_{0}$. Here $0<\delta_{\beta}<\beta-1$, as mentioned at the beginning of the paper. An important aspect for the second order expansion is that when reflecting the free boundary in the plane $T_{\lambda_{0}}$ (as defined in this paper) and when the plane is orthogonal to the free boundary at $x^{0}$, then

$$
u_{\lambda_{0}}-u=O\left(\left|x-x^{0}\right|^{2+\delta_{\beta}}\right),
$$

due to the symmetry of the first order term in the expansion (3.3).

In several symmetry problems a standard assumption imposed on the free boundary and the solution is that they both are $C^{2}$. This in particular implies that the solution has a second order expansion which is a polynomial of degree two, and from this expansion one can derive the so-called Serrin's boundary point lemma. This regularity assumption is actually much stronger than our second order asymptotic assumption, and in light of this and that our solutions have limited smoothness in the case $f(t) \approx t_{+}^{a}$ with $-1<a<0$ (they are $C^{1, \beta-1}$ ) one can see our secondorder asymptotic assumption as a realistic and natural assumption for treating this problem.

It needs to be remarked that informal computation and analysis (conveyed to us by John Andersson at KTH) points towards a plausible accurate linearization of solutions at regular free boundary points. Namely, we expect the following asymptotic expansion for solutions close to regular free boundary points

$$
u(x)=A_{0}\left(\left(x-x^{0}\right) \cdot \nu_{0}\right)_{+}^{\beta}+P_{2}\left(\left(x-x^{0}\right)-y\right) y^{\beta-1}+O\left(\left|x-x^{0}\right|^{\beta+1}\right),
$$

where $y=\left(\left(x-x^{0}\right) \cdot \nu_{0}\right) \nu_{0}$. We believe similar types of asymptotic expansions are to be expected at singular points. These are left for future to be explored. 


\section{REFERENCES}

[1] A. D. Alexandrov, A characteristic property of spheres, Ann. Mat. Pura Appl. (4) 58 (1962), 303-315, DOI 10.1007/BF02413056. MR.143162

[2] Mark Allen and Henrik Shahgholian, A new boundary Harnack principle (equations with right hand side), Arch. Ration. Mech. Anal. 234 (2019), no. 3, 1413-1444, DOI 10.1007/s00205019-01415-3. MR 4011700

[3] H. W. Alt and D. Phillips, A free boundary problem for semilinear elliptic equations, J. Reine Angew. Math. 368 (1986), 63-107. MR850615

[4] Agnid Banerjee and Bernd Kawohl, Overdetermined problems for the normalized p-Laplacian, Proc. Amer. Math. Soc. Ser. B 5 (2018), 18-24, DOI 10.1090/bproc/33. MR.3797009

[5] Leonardo Prange Bonorino, Regularity of the free boundary for some elliptic and parabolic problems. II, Comm. Partial Differential Equations 26 (2001), no. 3-4, 355-380, DOI 10.1081/PDE-100002572. MR 1842037

[6] B. Brandolini, C. Nitsch, P. Salani, and C. Trombetti, Serrin-type overdetermined problems: an alternative proof, Arch. Ration. Mech. Anal. 190 (2008), no. 2, 267-280, DOI 10.1007/s00205-008-0119-3. MR2448319

[7] Luis A. Caffarelli, Lavi Karp, and Henrik Shahgholian, Regularity of a free boundary with application to the Pompeiu problem, Ann. of Math. (2) 151 (2000), no. 1, 269-292, DOI 10.2307/121117. MR 1745013

[8] Andrea Cianchi and Paolo Salani, Overdetermined anisotropic elliptic problems, Math. Ann. 345 (2009), no. 4, 859-881, DOI 10.1007/s00208-009-0386-9. MR2545870

[9] Giulio Ciraolo, Rolando Magnanini, and Shigeru Sakaguchi, Symmetry of minimizers with a level surface parallel to the boundary, J. Eur. Math. Soc. (JEMS) 17 (2015), no. 11, 27892804, DOI 10.4171/JEMS/571. MR3420522

[10] Mariano Giaquinta and Enrico Giusti, Sharp estimates for the derivatives of local minima of variational integrals (English, with Italian summary), Boll. Un. Mat. Ital. A (6) 3 (1984), no. 2, 239-248, DOI 10.1016/s0294-1449(16)30429-2. MR753882

[11] B. Gidas, Wei Ming Ni, and L. Nirenberg, Symmetry and related properties via the maximum principle, Comm. Math. Phys. 68 (1979), no. 3, 209-243. MR.544879

[12] E. Lindgren and L. Silvestre, On the regularity of a singular variational problem, Unpublished manuscript, 2005.

[13] Erik Lindgren and Arshak Petrosyan, Regularity of the free boundary in a two-phase semilinear problem in two dimensions, Indiana Univ. Math. J. 57 (2008), no. 7, 3397-3417, DOI 10.1512/iumj.2008.57.3433. MR2492237

[14] Wolfgang Reichel, Radial symmetry for elliptic boundary-value problems on exterior domains, Arch. Rational Mech. Anal. 137 (1997), no. 4, 381-394, DOI 10.1007/s002050050034. MR.1463801

[15] James Serrin, A symmetry problem in potential theory, Arch. Rational Mech. Anal. 43 (1971), 304-318, DOI 10.1007/BF00250468. MR.333220

[16] Luis Silvestre and Boyan Sirakov, Overdetermined problems for fully nonlinear elliptic equations, Calc. Var. Partial Differential Equations 54 (2015), no. 1, 989-1007, DOI 10.1007/s00526-014-0814-x. MR3385189

[17] Eduardo V. Teixeira, Uniqueness, symmetry and full regularity of free boundary in optimization problems with volume constraint, Interfaces Free Bound. 9 (2007), no. 1, 133-148, DOI 10.4171/IFB/159. MR2317302

[18] Eduardo V. Teixeira, Regularity for the fully nonlinear dead-core problem, Math. Ann. 364 (2016), no. 3-4, 1121-1134, DOI 10.1007/s00208-015-1247-3. MR3466862

[19] Eduardo V. Teixeira, Nonlinear elliptic equations with mixed singularities, Potential Anal. 48 (2018), no. 3, 325-335, DOI 10.1007/s11118-017-9637-7. MR.3779091

Mathematics Division, American University in Dubai, Dubai, United Arab Emirates

Department of Mathematics, KTH Royal Institute of Technology, Stockholm, SweDEN 